\title{
Space Flight-Associated Neuroocular Syndrome, Idiopathic Intracranial Hypertension, and Pseudotumor Cerebri: Phenotypic Descriptions, Pathogenesis, and Hydrodynamics
}

\author{
Hassan Kesserwani ${ }^{1}$ \\ 1. Neurology, Flowers Medical Group, Dothan, USA
}

Corresponding author: Hassan Kesserwani, neuro1815@yahoo.com

\begin{abstract}
Recent data from astronauts who have returned to Earth from a long-duration space flight have unequivocally distinguished spaceflight-associated neuro-ocular syndrome (SANS) from idiopathic intracranial hypertension (IIH) and pseudotumor cerebri (PTC). We review the semiology and pathogenesis of these three entities, noting that optic disc edema is what unites them, and this where the similarities between SANS and IIH/PTC end. We distinguish between PTC and IIH and between SANS and IIH/PTC and review the medical and surgical therapy of IIH/PTC. The key to understanding the phenomenon of optic disc edema is the geometry of the optic nerve sheath, which is a simulacrum of an inverted Venturi tube. This allows us to theoretically study the hydrodynamics of the optic nerve sheath by applying simple physical laws, including the Venturi effect, Poiseuille's law, and Reynold's number, and we speculate on nature's design and the correlation of form and function in understanding how cerebrospinal fluid (CSF) circulates in the optic nerve sheath as it approaches the optic nerve head. Recent spectacular data on the histology of the blood nerve-barrier of the optic nerve disc and the glymphatic system of the optic nerve sheath will also help us understand the development of optic disc edema due to the microgravity-induced cephalad shift of CSF in SANS. We will explore the role of the sodium/potassium adenosine triphosphatase (ATPase) pump on choroid plexus epithelial cells and the aquaporin- 4 water receptors located on astrocyte end-feet and their complex interactions with the tetracyclines, mineralocorticoids, and therapeutic agents with carbonic anhydrase activity. We also adumbrate the complex interactions between obesity, vitamin A, and 11-beta-hydroxysteroid dehydrogenase and how the aquaporin-4 receptor relates to these interactions.
\end{abstract}

Review began 03/08/2021 Review ended 03/22/2021 Published 03/25/2021

() Copyright 2021 Kesserwani. This is an open access article distributed under the terms of the Creative Commons Attribution License CC-BY 4.0., which permits unrestricted use, distribution, and reproduction in any medium, provided the original author and source are credited.
Categories: Neurology, Ophthalmology

Keywords: idiopathic intracranial hypertension (iih), microgravity

\section{Introduction And Background}

We distinguish between spaceflight-induced neuroocular syndrome (SANS), idiopathic intracranial hypertension (IIH), and pseudotumor cerebri (PTC). The common denominator of SANS, IIH, and PTC is altered cerebrospinal fluid (CSF) hydrodynamics, the absence of a space-occupying lesion of the brain, the absence of ventriculomegaly, non-inflammatory CSF, and optic disc edema. SANS is due to altered CSF hydrodynamics induced by microgravity in outer space and its effect on the optic nerve disc; CSF pressure may be normal or mildly increased. PTC is due to raised CSF pressure induced by venous hypertension as seen with venous sinus thrombosis, an exogenous agent, such as hypovitaminosis A, hypervitaminosis A or tetracycline/minocycline, or other endocrine and neuro-humoral factors and their effects on the optic disc. $\mathrm{IIH}$ is also due to raised CSF pressure and occurs predominantly in younger obese women with no obvious immediate, proximate, endogenous, or exogenous etiology. Whether IIH/PTC is due to altered venous blood flow or altered CSF hydrodynamics or both is still debatable, with altered CSF hydrodynamics being the most likely cause. We will consider the terms CSF pressure and intracranial pressure (ICP) to be synonymous and apply them interchangeably. By hydrodynamics, we mean the pressure gradient across the lamina cribrosa of the optic disc, CSF pressure both intracranially and within the optic nerve sheath, and the dynamics of CSF flow along the optic nerve sheath. The distinguishing clinical features between IIH/PTC and SANS are listed in Table 1 [1]. 


\section{Cureus}

\begin{tabular}{|c|c|c|}
\hline & TERRESTRIAL IIH/PTC & SANS \\
\hline SEX & Ratio female-to-male is 9-to-1 & Equal frequency \\
\hline BODY HABITUS & Vast majority obese & Fit and athletic \\
\hline HEADACHES & Frequent and severe & Occasional and rare \\
\hline TRANSIENT VISUAL OBSCURATIONS & Frequent; almost three-quarter of patients & Never reported \\
\hline DIPLOPIA & One-third of patients & Never reported \\
\hline DISC EDEMA LATERALITY & Mostly bilateral & Mostly unilateral \\
\hline CSF PRESSURE & Increased & Normal or mildly increased \\
\hline CHOROIDAL FOLDS & Concentric around optic nerve head & Linear folds \\
\hline
\end{tabular}

\section{TABLE 1: Distinguishing between IIH/PTC and SANS}

idiopathic intracranial hypertension (IIH), pseudotumor cerebri (PTC), spaceflight-associated neuroocular syndrome (SANS)

Why SANS is mostly unilateral is not entirely clear, and the authors do not offer any explanation. It may have to do with local hydrodynamics and trabeculation/septation of the optic nerve sheath, as outlined in the Discussion section. Visual field testing in SANS revealed enlargement of the blind spot or scotomas. The nature of the scotomas was not identified in the report [1].

Walter Dandy published a series of 22 patients that had symptoms of high CSF pressure (headaches, transient visual obscurations, pulsatile tinnitus, diplopia), absence of tumor by ventriculography, and high CSF pressure with a lumbar puncture. All patients were decompressed by a subtemporal craniotomy with favorable long-term results. The absence of ventricular dilation suggested to him a lack of a CSF flow problem. He also made the astute observation that the chronicity of this condition precludes cerebral edema as a cause of this condition. The rapid relief with decompressive surgery was explained by invoking the Monroe-Kelley doctrine, that the drop in ICP reflects a drop in intracranial blood volume. He diagnosed these cases as " high intracranial pressure without a brain tumor," analogous to what we refer to now as pseudotumor cerebri [2].

The modified Dandy criteria are as follows: 1) a lumbar puncture confirming a high CSF pressure, greater than 200-250 mmHg in an adult and $280 \mathrm{mmHg}$ in a child; 2) non-inflammatory CSF; 3) symptoms and signs concurrent with high CSF pressure with at least one of a) pulse-synchronous tinnitus, b) sixth nerve palsy, c) papilledema d) partially empty sella or distended or tortuous optic nerve sheath, e) magnetic resonance venous (MRV) with transverse venous sinus collapse or stenosis; 4) non-focal neurological signs except for a sixth nerve palsy; 5) no space-occupying lesion on magnetic resonance imaging (MRI) of the brain and no structural deformity of the ventricular system obstructing the flow of CSF; 6) funduscopic evidence of papilledema; 7) MRV imaging excluding a venous sinus thrombosis [3].

Friedmann DI et al. propose that these criteria be relaxed in patients with typical symptoms and signs of IIH/PTC but without papilledema especially in children and in adults early in the disease who may not register a high CSF pressure. This may be accounted for by subtle optic atrophy or fibrosis of the optic nerve fiber layer and may account for cases of obese women who have chronic daily persistent headaches [4].

The symptom profile of PTC/IIH reflects the complex hemodynamics of increased CSF pressure and its local effects (Table 2). 


\section{Cureus}

\begin{tabular}{|c|c|c|c|}
\hline & SYMPTOM & $\begin{array}{l}\text { INCIDENCE } \\
(\%)\end{array}$ & MECHANISM \\
\hline $\begin{array}{l}\text { Weisberg LA } \\
{[5]}\end{array}$ & Headache & 99 & Stretch of meninges, worse with supine posture (hydrostatic) and Valsalva maneuver \\
\hline $\begin{array}{l}\text { Giuseffi V, et } \\
\text { al. [6] }\end{array}$ & $\begin{array}{l}\text { Transient } \\
\text { visual } \\
\text { obscurations }\end{array}$ & 68 & Triggered by posture/transient ischemia of optic disc due to optic nerve swelling \\
\hline Wall M [7] & $\begin{array}{l}\text { Horizontal } \\
\text { diplopia }\end{array}$ & 38 & $\begin{array}{l}\text { Neuropraxia of the sixth cranial nerve due to high ICP and compression as it exits } \\
\text { Dorello's canal }\end{array}$ \\
\hline Smith JL [8] & $\begin{array}{l}\text { Pulsatile } \\
\text { tinnitus }\end{array}$ & 58 & $\begin{array}{l}\text { Resonance of arterial pulsations with venous pulsations due to venous hypertension or } \\
\text { turbulent flow through stenosis of the transverse sinus. Improves with compression of } \\
\text { ipsilateral internal jugular vein }\end{array}$ \\
\hline $\begin{array}{l}\text { Kleinschmidt } \\
\text { KK, et al. [9] }\end{array}$ & $\begin{array}{l}\text { Retrobulbar } \\
\text { pain with eye } \\
\text { motion }\end{array}$ & 44 & Due to swelling of optic nerve sheath \\
\hline $\begin{array}{l}\text { Friedmann } \\
\text { DI, et al. [10] }\end{array}$ & Vision loss & $30-35$ & See table 4 \\
\hline $\begin{array}{l}\text { Digre KB, et } \\
\text { al. [11] }\end{array}$ & $\begin{array}{l}\text { Cervical } \\
\text { radiculopathy }\end{array}$ & 19 & Increased CSF pressure in radicular nerve root sheath \\
\hline
\end{tabular}

\section{TABLE 2: The symptoms of IIH/PTC, their incidence, and the putative mechanism of action}

pseudotumor cerebri (PTC), idiopathic intracranial hypertension (IIH), intracranial pressure (ICP), cerebrospinal fluid (CSF)

Morphological changes induced by high CSF pressure on the eye globe (retrolaminar space), optic nerve sheath, and brain reflect both global and local hemodynamic changes (Table 3) [12-13].

\begin{tabular}{|l|c|c|}
\hline MORPHOLOGICAL FINDING & MAXIMUM SENSITIVITY (\%) & SPECIFICITY (\%) \\
\hline Empty sella & 26.7 & 94.6 \\
\hline Slit-like ventricles & 3.3 & 100 \\
\hline Enlarged optic nerve sheath & 66.7 & 100 \\
\hline Bulging of the optic disc into eye-globe & 30 & 98.1 \\
\hline Optic nerve enhancement & 50 & 91.1 \\
\hline Tortuosity of the optic nerve & 40 & 100 \\
\hline Flattening of lamina cribrosa & 80 & 93 \\
\hline Transverse sinus narrowing & 93 & Unknown \\
\hline Cerebellar ectopia (more than $5 \mathrm{~mm}$, peg-like) & 21.9 & \\
\hline
\end{tabular}

TABLE 3: Morphological changes of high CSF pressure locally and globally on the eye-globe, optic nerve sheath, and brain

cerebrospinal fluid (CSF), millimeter (mm)

Papilledema reflects an increase in CSF pressure. Optic nerve swelling begins in the lower pole and progresses to the upper pole and then the nasal pole and reaches a climax in disc margin blurring and optic disc edema. There is concomitant venous distension and a loss of venous pulsation [14]. Interestingly, the papillomacular bundle is spared until late in the disease [15]. In late papilledema, there are retinal nerve fiber infarcts due to venous hypertension, leading to cotton-wool spots [16]. The effects of increased CSF 


\section{Cureus}

pressure on the optic disc and retinal nerve fiber layer (RNFL) include (Table 4) [17].

\section{VISUAL FIELD DEFECT AND RNFL ABNORMALITIES}

Arcuate-shaped

Enlargement of the blind spot

Choroidal folds

Peri-papillary hemorrhages

Deep hemorrhages

Star figure

Subretinal macular edema

Subretinal neovascular membrane
MECHANISM AND COMMENTARY

Mostly inferior periphery with more marked loss of nerve fibers in the superior retina (thinner layer superior optic disc, with less glial support)

Accumulation of peri-papillary sub-retinal fluid

Bulging of optic nerve sheath into eye-globe with crests and troughs of the choroidal layer or wrinkling of the retinal pigment epithelium

Leaky retinal arteries

Deep to retinal pigment epithelium; poor outcome

Exudate: protein and lipid leakage from retinal capillaries between fovea and disc

$10 \%$ with severe papilledema

Rapid vision loss

TABLE 4: Effects of increased ICP on the optic disc and RNFL

intracranial pressure (ICP), retinal nerve fiber layer (RNFL)

The mainstay of therapy is weight loss and the use of carbonic anhydrase inhibitors. The latter reduce CSF production and induce diuresis [18-19]. The older carbonic anhydrase inhibitor methazolamide was also effective. Though loop diuretics, bumetanide and furosemide, are less effective, they have some benefit [20]. Topiramate also works through its weight-loss action and carbonic anhydrase inhibition [21]. Further commentary on the details and recently discovered novel mechanisms of action of pharmacological agents will be outlined in the Discussion section.

Should medical therapy fail, surgical therapy falls into three categories: CSF diversion procedures, bariatric surgery, and venous stenting. A summary of the procedures and main principles and noteworthy points are listed below (Table 5). 


\section{Cureus}

\section{CSF DIVERSION}

PROCEDURE OR

OTHER SURGERY

Dandy

DW [1]

Sub-cranial

craniectomy (flap)

Al-

Saadany Lumbar-peritoneal

WF, et al. shunt

[22]

McGirt

MJ, et al.

[23]

Lee MC,

et al. [24]

Banta JT, Optic nerve sheath

et al [25] fenestration

Johnston

I, et al.

[26]

Bussiere

M, et al. Venous sinus stenting

[27]

Sugerman

$\mathrm{HJ}$, et al. Bariatric surgery

[28]
COMMENT

Dandy noted a rapid drop in ICP and ascribed it to arterial pressure and blood volume

Subarachnoid / $80 \%$ success rate / frequent revision/lower infection rate than ventriculoperitoneal shunt

Difficult placement as many patients have a slit-like ventricle but less shunt failure than lumboperitoneal shunts

When lumbar drainage and slit-like ventricles are not accessible

Slit in optic nerve sheath; may create a fistula or cut trabeculae allowing the release of retrolaminar pressure. Highly effective. Fenestration of one eye may also help the other eye via continuity of trabecular meshwork.

Short term: pregnancy, cryptococcal meningitis. Otherwise, high failure rate of $66 \%$

High risk of complications and requirement of long-term dual antiplatelet therapy. Asymmetry in transverse sinuses as high as $73 \%$ in the general population. However, effective therapy

Highly effective. Weight loss reduced CSF pressure to normal

TABLE 5: List of surgical procedures and their risk-benefit profile

intracranial pressure (ICP), cerebrospinal fluid (CSF)

SANS in a long-duration space flight leads to decreased near-vision; otherwise, astronauts are asymptomatic. Optic disc edema, eye-globe flattening, choroidal folds, and cotton-wool spots mimic what happens in terrestrial IIH. Globe flattening, which is the loss of curvature (convexity) of the posterior sclera, leads to the shortening of the globe and anterior displacement of the fovea, with a subsequent hyperopic shift of the refractive index. However, CSF pressure via lumbar puncture (LP) measurement is normal or mildly elevated post-flight. In microgravity, it is postulated that there is a cephalad shift in CSF leading to internal jugular vein distension and reduced CSF drainage into the venous system. This may lead to locally increased ICP and subsequent distension of the optic nerve sheath, stasis of axoplasmic flow, and disc edema. Surprisingly, despite the development of an empty sella, none of the astronauts developed any significant symptoms of increased ICP such as headaches, tinnitus, diplopia, or transient visual obscurations. The optic disc edema may last for six months post-mission [29]. Why the astronauts do not develop symptoms of increased ICP may be related to the fact that optic disc edema is due to local hydrodynamic changes at the optic nerve sheath and not global effects of increased ICP with stretching of the meninges and traction of the cranial nerves.

Interestingly, head-down tilting and prolonged bed rest can mimic microgravity-induced internal jugular venous distension and thickening of the retinal nerve fiber layer of the optic nerve on ocular computerized tomography (OCT), with thickening of the retinal fiber layer with OCT being an early marker of optic disc edema [30]. Increased ICP leads to elevated hypoxia-inducible factor 1-alfa (HIF-1 alfa) in the retinal ganglion cells (RGC) and leakage of the blood-optic nerve barrier with a spillover of fluid and protein in the optic nerve head and subretinal space in the papilla. Increased ICP may also lead to the increased stiffness of the trabecular meshwork in the optic nerve sheath increasing local CSF pressure [31-32]. The CSF may also become loculated within the optic nerve sheath explaining the persistent optic disc edema despite return to Earth in SANS and post-lumbar decompression in terrestrial IIH [33].

\section{Review}




\section{Anatomy of the optic nerve sheath and pressure differentials}

The optic nerve sheath is divided into three segments: the bulbar segment, the widest part, adjacent to the eye globe; and the intraorbital and intracanalicular segments. This tube is not necessarily a simple continuous tube with laminar flow (Figure 1).

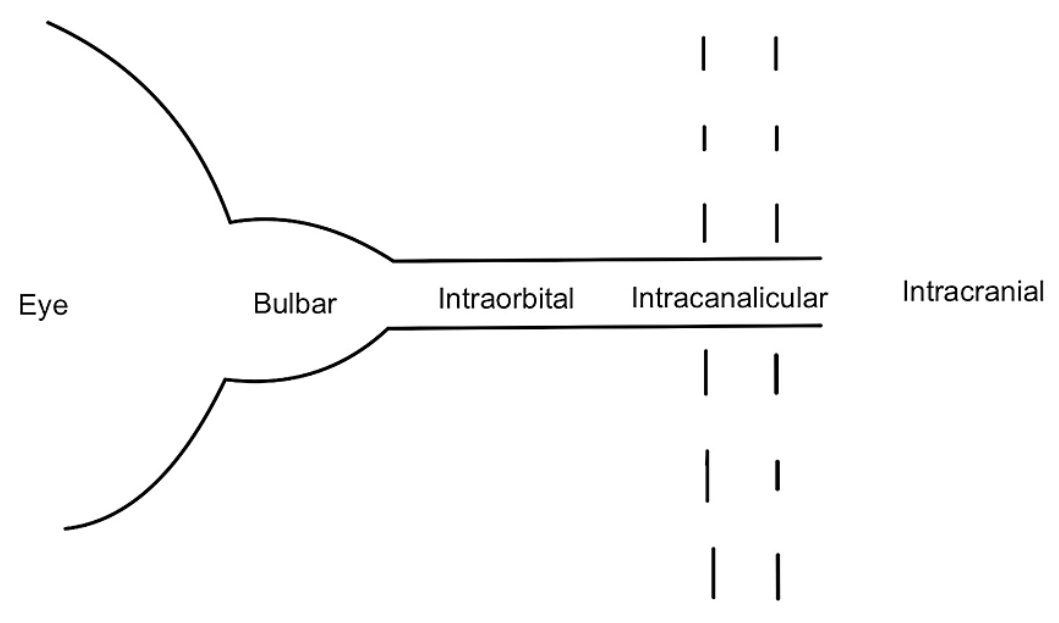

FIGURE 1: Optic nerve sheath subdivisions: bulbar, intraorbital, and canalicular segments

Instead, between the pia and arachnoid layers, there is a fine trabeculated meshwork in the bulbar segment, loculation by septa and pillars in the intraorbital segment, and by trabeculae and pillars in the intracanalicular segment. This reticulated pattern is more like a complex web or a labyrinth where CSF reaches a dead-end at the eye-globe. CSF from the cisterns of the brain are pumped into this cavernous system almost like a hydraulic pump. Therefore, the optic disc is defended by the scleral lamina cribrosa, which is at the mercy of a pressure gradient between the intraocular pressure (IOP) and the ICP, where locally at the bulbar segment, the CSF pressure is referred to as the retrolaminar pressure (RLP). In glaucoma, IOP exceeds RLP and the optic disc is cupped into the retrobulbar space. When the RLP exceeds the IOP, the optic disc bulges into the eye-globe. Both scenarios alter the morphology of the lamina cribrosa, with compression leading to ischemia, retardation of axoplasmic flow, and neural degeneration of the retinal ganglion cells [34].

\section{Hydrodynamics of the optic nerve sheath: Venturi effect, Poiseuille's law, and Reynold's number}

Why is the optic nerve sheath wider at the bulbar end? To answer this question, we need to return to the basics of hydrodynamics. When describing the flow of a fluid, we use the parameters of pressure $p$, density $\rho$, velocity $v$, and the volume flow per unit time $\frac{d V}{d t}$ across a cross-sectional area $A$. A Venturi pipe is one where the cross-sectional area decreases and a reverse Venturi pipe is one where the cross-sectional area increases as we travel along the pipe (Figure 2).

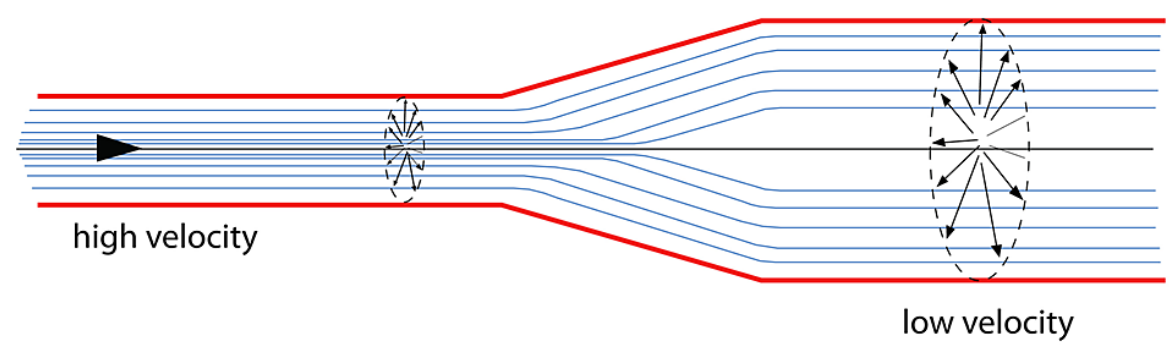

\section{FIGURE 2: Reverse Venturi tube}

When moving from a lower cross-sectional area of the tubing to a higher cross-section area, the velocity of fluid flow decreases and the pressure head increases. 
Assuming the net flow of fluid volume across any section of the tube is a constant, we have the simple differential equation:

$$
\frac{d V}{d t}=\frac{A \cdot v}{t}=\text { constant }
$$

This tells us that the velocity of flow $v$ at any point along the tube is inversely proportional to the crosssectional area $A$. Therefore, the velocity drops at the wider end of the reverse Venturi tube.

To explain what happens to the pressure $p$, we deploy Poiseuille's Law, which states:

$$
\frac{d V}{d t}=\frac{\pi p r^{4}}{8 \eta l}
$$

where $r$ is the radius of the tube, $l$ is the length, and $\eta$ is the viscosity of the fluid. There is an inverse relationship between the radius $r$ of the tube and the pressure $p$ at any point [35].

In summary, as we approach the bulbar end of the optic nerve sheaths, theoretically, two physical events happen, the CSF pressure increases and the speed of flow decreases. This actually follows from the Law of Conservation of Energy, where the total energy of a system (potential and kinetic energy) is conserved.

$$
\frac{m p}{\rho}+\frac{m v^{2}}{2}=0
$$

Again, we see that the pressure $p$ is inversely related to the velocity $v$ at any given point. This is nature's way of maintaining a pressure head when confronted with narrow bored tunnels as in the optic nerve sheath to allow CSF to bathe the optic nerve along its whole length.

How does one explain the septation and trabeculation of the optic nerve sheath? Here, we need to understand the concepts of laminar flow and turbulent flow. We believe that the septation and trabeculation of the optic nerve sheath induce turbulent flow when the ICP rises to high enough levels. This blunts the pressure head and protects the optic nerve sheath and head. To understand this, we need to know what Reynold's number is. Reynolds's number $R$ is the ratio of inertial resistance/viscous resistance expressed mathematically as:

$$
R=\frac{\rho v l}{\eta}
$$

When Reynold's number $R$ is low, the flow is steady, smooth, and laminar. When $R$ is high, the fluid flow is determined by the momentum more than the viscosity and the fluid flow is turbulent (Figure 3) [35].

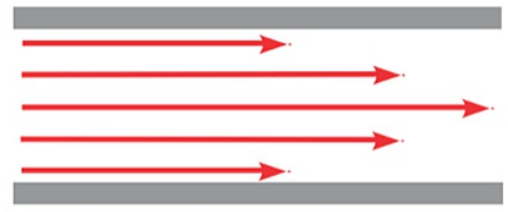

Laminar Flow

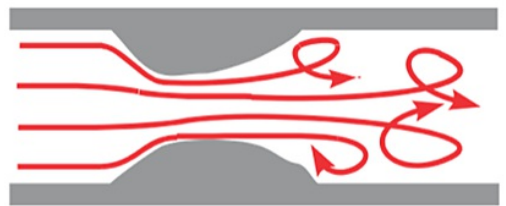

Turbulent Flow

\section{FIGURE 3: Laminar flow versus turbulent flow}

The septations and trabeculations create eddies and turbulent flow when the CSF pressure increases significantly, converting a laminar flow to a turbulent flow and reducing the pressure head at the optic nerve sheath (Figure 4). 


\section{Cureus}

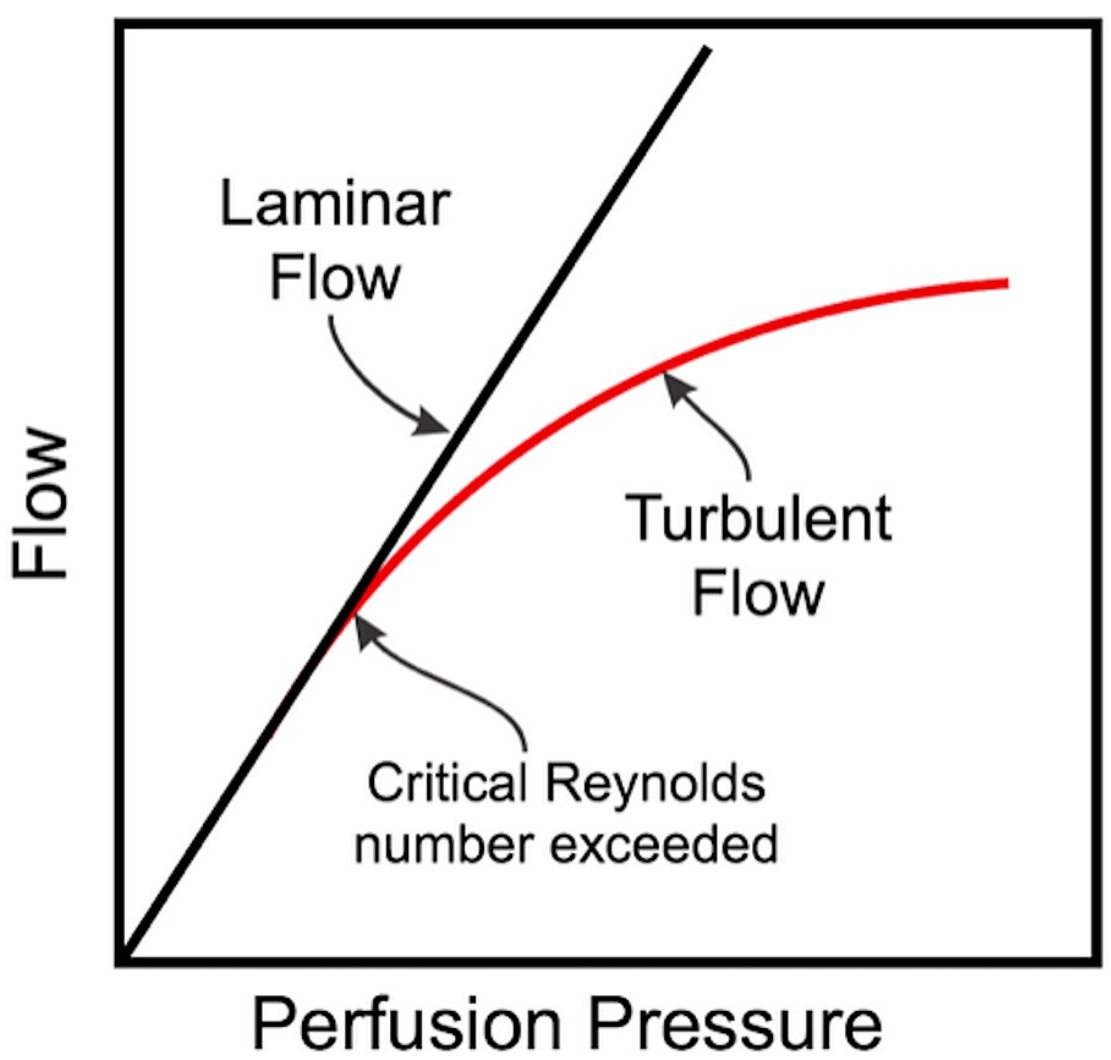

FIGURE 4: Flow-perfusion pressure diagram

Note the drop in perfusion pressure when the flow is turbulent.

These purely theoretical considerations are supported by experimental data. First, we know that CSF pressure determines the retrolaminar tissue pressure in the optic nerve sheath subarachnoid space based upon micropipette-pressure transducer recordings in an anesthetized dog [36]. Second, using

microcomputerised scanning of porcine eyes, high CSF pressures correlated with deformation and distortion of the lamina cribrosa even with normal intraocular pressure [37]. Third, a remarkable study correlating optic nerve sheath diameter (a correlate of local CSF pressure) using transcranial Doppler and lumbar CSF pressure obtained a linear relationship between both parameters but with different slopes for different patients. The linear relationship occurred between 15 and $40 \mathrm{mmHg}$. After this, the optic nerve sheath reaches its maximum elasticity and yields, or based upon our hypothesis, the CSF flow reaches its Reynold's number and we produce turbulence and a drop in CSF pressure [38].

\section{The role of aquaporins and a possible pathway for tetracycline pathology in PTC}

CSF is produced by the choroid plexus (two-thirds of the volume) and the ependymal lining of the ventricles (one-third of the volume) with a gradual decline in the daily total volume with aging. Aquaporin-4 receptors on astrocyte end-feet facilitate the flow of water from the choroid plexus and ependymal epithelia into the CSF. There is also direct pumping of sodium into the CSF by the sodium/potassium adenosine triphosphates (ATPase) pump and the enzyme carbonic anhydrase that catalyzes the reaction between a carbon dioxide molecule and a water molecule to produce a proton and bicarbonate ions. The electrochemical gradient pushes protons and sodium ions along with water molecules into the CSF and drainage into the venous sinuses is across the arachnoid granulations and into the nasal lymphatics across the cribriform plate. The carbonic anhydrase activity inhibitors, acetazolamide, topiramate, and zonisamide have all been shown to inhibit aquaporin-4 activity. Nevertheless, the evidence for CSF overproduction in PTC/IIH is limited [39].

After injury or inflammation, activated astrocytes release matrix metalloproteinase-9 (MMP-9), which disrupts the basal lamina between the astrocyte end-feet and the endothelial cells. This leads to a breakdown in the blood-brain barrier and vasogenic edema facilitated by the upregulation of aquaporin- 4 receptors. Adult rats subjected to traumatic brain injury developed less blood-brain barrier disruption with less upregulation of MMP-9 and aquaporin-4 receptors after injection with minocycline, an MMP-9 inhibitor. 
This was a beneficial effect but nevertheless provides evidence whereby the tetracyclines can modulate aquaporin- 4 receptors and potentially lead to excessive CSF production [40]. Whether the tetracyclines act differently in non-injured brains and whether this is genetically determined is a complex question worthy of further study.

\section{Obesity, inflammation, and 11-beta-hydroxysteroid dehydrogenase in IIH}

Obesity and rapid weight gain in women are unequivocally very strongly associated with IIH, implying a major role for the neuroendocrine role of adiposity [41]. Even a less severe weight loss of $6 \%$ is a more potent catalyst for the resolution of papilledema than pharmacotherapy with acetazolamide [42]. Abdominal obesity may increase abdominal pressure and central venous pressure with secondary venous distension of the internal jugular vein and increased intracranial pressure. However, the association of CSF pressure (lumbar puncture) and body mass index (BMI) has a weak positive correlation. Obstructive sleep apnea increases ICP by increasing intrathoracic pressure and internal jugular vein distension and by hypoxia leading to hypercapnia with secondary vasodilatation.

Obesity is associated with elevated levels of serum and adipose 11-beta-hydroxysteroid dehydrogenase levels (11-beta-HSD). 11-beta-HSD regulates cortisol action intracellularly and increases the activity of the sodium/potassium ATPase pump, increasing the production of CSF in the choroid plexus epithelium. A fall in serum and CSF 11-beta-HSD levels correlated with a drop in CSF pressure and weight loss in a group of 25 obese patients with IIH [43]. There was a benefit of 11-beta-HSD inhibitors in a small phase two study of 31 obese women with IIH. The mean drop of CSF pressure was $4.3 \mathrm{~cm}$ water and the agent was safe and welltolerated [44].

\section{Obesity, polycystic ovarian syndrome (PCOS), vitamin A, and IIH}

Adipose tissue contains the enzyme aromatase, which converts the androgen androstenedione to estrogens. Adipose tissue contains the enzyme aromatase, which converts the androgen androstenedione to estrogens. Estrogens are known to influence aquaporin-4 receptors and retinoic acid synthesis (see below) [45].

In IIH, the androgen profile is different from that in PCOS, despite the fact that PCOS is more prevalent in IIH than in the general population. In IIH, serum and CSF testosterone are increased and their precursors dehydroepiandrosterone (DHEAS) and androstenedione - are decreased as compared to in PCOS and simple obesity. Testosterone is known to drive the choroidal plexus sodium/potassium ATPase, potentially increasing CSF output. The precursors DHEAS and androstenedione are the main circulating androgens in PCOS leading to hirsutism and android features, not seen in IIH [46].

Adipose tissue also increases the production of active vitamin A, retinoic acid, from the pro-hormone retinal. Both hypervitaminosis A and hypovitaminosis A can lead to IIH/PTC. Hypovitaminosis A can lead to fibrosis of the arachnoid villi in calves and impaired CSF absorption [47].

\section{Microgravity-induced cephalad shift of fluid, local optic nerve sheath blood-nerve barrier defect, glymphatic flow, and SANS}

SANS is a disease of the optic nerve head. As we outlined earlier, lumbar punctures performed post-long duration flight in astronauts have shown normal or mildly elevated CSF pressures. The cephalad shift of fluid in a microgravity environment leads to internal jugular vein distension and a puffy face. All astronauts develop optic nerve fiber layer thickening of the optic nerve head when visualized with OCT while $15 \%$ of astronauts develop papilledema. Prolonged head-down tilt table posture can replicate these findings in the laboratory [48]. This pressure head facilitates the exudation of interstitial fluid in the pre-laminar region of the optic nerve head, where the blood-nerve barrier has been shown to be "leaky " as compared to elsewhere in the retina [49].

The glymphatic system is a paravascular channel pathway delimited by astroglial endfeet that allows intravitreal and retinal fluid to flow posteriorly into the optic nerve sheath subarachnoid space. The translaminar cribrosa pressure, which is equal to the intraocular pressure minus the ICP is about $4 \mathrm{mmHg}$. In microgravity, with the cephalad shift of CSF, this pressure differential is reversed and fluid is pressureforced into the pre-laminar optic disc with the formation of optic disc edema. This idea is not far-fetched, as, in certain instances of subarachnoid hemorrhage, blood finds its way into the vitreous of the eye (Terson syndrome) [50].

\section{Conclusions}

As spaceflight becomes increasingly common, the National Aeronautics Space Administration (NASA) has made SANS a top priority. Up to $15 \%$ of astronauts will develop optic disc edema and mitigating strategies, such as veno-occlusive thigh compression devices, are being investigated to counteract the cephalad shift of body fluids during space travel. As space tourists and travelers become commonplace, knowledge of SANS will become a prerequisite for the practicing clinician. In this article, we have distinguished between the 
clinical features and pathophysiology of SANS and IIH/PTC. We delve deeper into the hydrodynamics of the optic nerve sheath and the unique histological features of the lamina cribrosa and the glymphatic system, where we emphasized the role of translaminar pressure in the pathogenesis of optic disc edema. The complex interactions between obesity, vitamin A, the steroidogenic enzyme 11-beta-hydroxysteroid dehydrogenase, the aquaporin- 4 receptor of astrocytes, and the sodium/potassium ATPase pump on choroid plexus epithelial cells and the generation of optic disc edema were explored. Finally, we draw attention to the non-traditional and novel mechanisms of action of the pharmacological agents used to treat PTC/IIH. This article is one of the earliest articles to address SANS in a non-space science-related journal.

\section{Additional Information}

\section{Disclosures}

Conflicts of interest: In compliance with the ICMJE uniform disclosure form, all authors declare the following: Payment/services info: All authors have declared that no financial support was received from any organization for the submitted work. Financial relationships: All authors have declared that they have no financial relationships at present or within the previous three years with any organizations that might have an interest in the submitted work. Other relationships: All authors have declared that there are no other relationships or activities that could appear to have influenced the submitted work.

\section{References}

1. Lee AG, Mader TH, Gibson CR, et al.: Spaceflight associated neuro-ocular syndrome (SANS) and the neuroophthalmologic effects of microgravity: a review and an update. NPJ Microgravity. 2020, 6:7. 10.1038/s41526-020-0097-9

2. Dandy WE: Intracranial pressure without brain tumor: diagnosis and treatment . Ann Surg. 1937, 106:492513. 10.1097/00000658-193710000-00002

3. Wall M, Corbett JJ: Revised diagnostic criteria for the pseudotumor cerebri syndrome in adults and children . Neurology. 2014, 83:198-9. 10.1212/01.wnl.0000452039.32455.3e

4. Friedman DI, Liu GT, Digre KB: Revised diagnostic criteria for the pseudotumor cerebri syndrome in adults and children. Neurology. 2013, 81:1159-65. 10.1212/WNL.0b013e3182a55f17

5. Weisberg LA: Benign intracranial hypertension. Medicine (Baltimore). 1975, 54:197-207. 10.1097/00005792197505000-00002

6. Giuseffi V, Wall M, Siegel PZ, Rojas PB: Symptoms and disease associations in idiopathic intracranial hypertension (pseudotumor cerebri): a case-control study. Neurology. 1991, 41:239-44. 10.1212/WNL.41.2_Part_1.239

7. Wall M: Idiopathic intracranial hypertension. Neurol Clin. 2010, 28:593-617. 10.1016/j.ncl.2010.03.003

8. Smith JL: Whence pseudotumor cerebri?. J Clin Neuroophthalmol. 1985, 5:55-56.

9. Kleinschmidt JJ, Digre KB, Hanover R: Idiopathic intracranial hypertension: relationship to depression, anxiety, and quality of life. Neurology. 2000, 54:319-24. 10.1212/WNL.54.2.319

10. Friedman DI, Jacobson DM: Idiopathic intracranial hypertension. J Neuroophthalmol. 2004, 24:138-45. 10.1097/00041327-200406000-00009

11. Digre KB, Bruce BB, McDermott MP, Galetta KM, Balcer LJ, Wall M, NORDIC Idiopathic Intracranial Hypertension Study Group: Quality of life in idiopathic intracranial hypertension at diagnosis: IIH Treatment Trial results. Neurology. 2015, 84:2449-2456.

12. Degnan AJ, Levy LM: Pseudotumor cerebri: brief review of clinical syndrome and imaging findings . AJNR Am J Neuroradiol. 2011, 32:1986-1993. 10.3174/ajnr.A2404

13. Aiken AH, Hoots JA, Saindane AM, Hudgins PA: Incidence of cerebellar tonsillar ectopia in idiopathic intracranial hypertension: a mimic of the Chiari I malformation. AJNR Am J Neuroradiol. 2012, 33:19011906. 10.3174/ajnr.A3068

14. Hayreh MS, Hayreh SS: Optic disc edema in raised intracranial pressure. I. Evolution and resolution . Arch Ophthalmol. 1977, 95:1237-1244. 10.1001/archopht.1977.04450070135013

15. Wall M, George DN: Visual loss in pseudotumor cerebri. Incidence and defects related to visual field strategy. Arch Neurol. 1987, 44:170-175. 10.1001/archneur.1987.00520140040015

16. McLeod D: Why cotton wool spots should not be regarded as retinal nerve fibre layer infarcts . Br J Ophthalmol. 2005, 89:229-37. 10.1136/bjo.2004.058347

17. Schirmer CM, Hedges TR 3rd: Mechanisms of visual loss in papilledema . Neurosurg Focus. 2007, 23:E5. 10.3171/FOC-07/11/E5

18. McCarthy KD, Reed DJ: The effect of acetazolamide and furosemide on cerebrospinal fluid production and choroid plexus carbonic anhydrase activity. J Pharmacol Exp Ther. 1974, 189:194-201.

19. Kesler A, Hadayer A, Goldhammer Y, Almog Y, Korczyn AD: Idiopathic intracranial hypertension: risk of recurrences. Neurology. 2004, 63:1737-1739. 10.1212/01.wnl.0000143067.40281.16

20. Kahle KT, Walcott BP, Staley KJ: Resolution of headache and papilledema in idiopathic intracranial hypertension associated with inhibition of $\mathrm{Na}+-\mathrm{K}+-2 \mathrm{Cl}$ - cotransport. J Child Neurol. 2011, 26:205-8. $10.1177 / 0883073810391264$

21. Celebisoy N, Gökçay F, Sirin H, Akyürekli O: Treatment of idiopathic intracranial hypertension: topiramate vs acetazolamide, an open-label study. Acta Neurol Scand. 2007, 116:322-7. 10.1111/j.16000404.2007.00905.x

22. El-Saadany WF, Farhoud A, Zidan I: Lumboperitoneal shunt for idiopathic intracranial hypertension: patients' selection and outcome. Neurosurg Rev. 2012, 35:239-44. 10.1007/s10143-011-0350-5

23. McGirt MJ, Woodworth G, Thomas G, Miller N, Williams M, Rigamonti D: Cerebrospinal fluid shunt placement for pseudotumor cerebri-associated intractable headache: predictors of treatment response and an analysis of long-term outcomes. J Neurosurg. 2004, 101:627-32. 10.3171/jns.2004.101.4.0627 
24. Lee MC, Yamini B, Frim DM: Pseudotumor cerebri patients with shunts from the cisterna magna: clinical course and telemetric intracranial pressure data. Neurosurgery. 2004, 55:1094-9. 10.1227/01.NEU.0000141314.28782.10

25. Banta JT, Farris BK: Pseudotumor cerebri and optic nerve sheath decompression. Ophthalmology. 2000, 107:1907-1912. 10.1016/S0161-6420(00)00340-7

26. Johnston I, Paterson A, Besser M: The treatment of benign intra- cranial hypertension: a review of 134 cases. Surg Neurol. 1981, 16:218-224. 10.1016/0090-3019(81)90010-0

27. Bussière M, Falero R, Nicolle D, Proulx A, Patel V, Pelz D: Unilateral transverse sinus stenting of patients with idiopathic intracranial hypertension. AJNR Am J Neuroradiol. 2010, 31:645-50. 10.3174/ajnr.A1890

28. Sugerman HJ, Felton WL 3rd, Salvant JB Jr, Sismanis A, Kellum JM: Effects of surgically induced weight loss on idiopathic intracranial hypertension in morbid obesity. Neurology. 1995, 45:1655-9. 10.1212/wnl.45.9.1655

29. Mader TH, Gibson CR, Pass AF, et al.: Optic disc edema, globe flattening, choroidal folds, and hyperopic shifts observed in astronauts after long-duration space flight. Ophthalmology. 2011, 118:2058-69. 10.1016/j.ophtha.2011.06.021

30. Taibbi G, Cromwell RL, Zanello SB, Yarbough PO, Ploutz-Snyder RJ, Godley BF, Vizzeri G: Ocular outcomes comparison between 14- and 70-day head- down-tilt bed rest. Invest Ophthalmol Vis Sci. 2016, 57:495-501. 10.1167/iovs.15-18530

31. Shen G, Link S, Kumar S, et al.: Characterization of retinal ganglion cell and optic nerve phenotypes caused by sustained intracranial pressure elevation in mice. Sci Rep. 2018, 8:2856. 10.1038/s41598-018-21254-8

32. Mader TH, Gibson CR, Otto CA, et al.: Persistent asymmetric optic disc swelling after long-duration space flight: Implications for pathogenesis. J Neuroophthalmol. 2017, 37:133-139. 10.1097/WNO.0000000000000467

33. Killer HE, Subramanian PS: Compartmentalized cerebrospinal fluid. Int Ophthalmol Clin. 2014, 54:95-102. 10.1097/IIO.0000000000000010

34. Killer HE, Laeng HR, Flammer J, Groscurth P: Architecture of arachnoid trabeculae, pillars, and septa in the subarachnoid space of the human optic nerve: anatomy and clinical considerations. Br J Ophthalmol. 2003, 87:777-81. 10.1136/bjo.87.6.777

35. Rubinow SI: Introduction to Mathematical Biology. Rubinow SI (ed): Dover Publications, United States; 1975.

36. Morgan WH, Dao-Yi Y, Cooper RL, Alder VA, Cringle SJ, Constable IJ: The influence of the cerebrospinal fluid pressure on the lamina cribrosa tissue pressure gradient. Invest Ophthalmol Vis Sci. 1995, 36:1163-72.

37. Feola AJ, Coudrillier B, Mulvihill J, et al.: Deformation of the lamina cribrosa and optic nerve due to changes in cerebrospinal fluid pressure. Invest Ophthalmol Vis Sci. 2017, 58:2070-2078. 10.1167/iovs.16-21393

38. Hansen HC, Helmke K: Validation of the optic nerve sheath response to changing cerebrospinal fluid pressure: ultrasound findings during intrathecal infusion tests. J Neurosurg. 1997, 87:34-40. 10.3171/jns.1997.87.1.0034

39. McGeeney BE, Friedman DI: Pseudotumor cerebri pathophysiology. Headache. 2014, 54:445-458. 10.1111/head.12291

40. Higashida T, Kreipke CW, Rafols JA, et al.: The role of hypoxia-inducible factor- $1 \alpha$, aquaporin-4, and matrix metalloproteinase-9 in blood-brain barrier disruption and brain edema after traumatic brain injury. J Neurosurg. 2011, 114:92-101. 10.3171/2010.6.JNS10207

41. Daniels AB, Liu GT, Volpe NJ, et al.: Profiles of obesity, weight gain, and quality of life in idiopathic intracranial hypertension (pseudotumor cerebri). Am J Ophthalmol. 2007, 143:635-41. 10.1016/j.ajo.2006.12.040

42. Johnson LN, Krohel GB, Madsen RW, March GA Jr: The role of weight loss and acetazolamide in the treatment of idiopathic intracranial hypertension (pseudotumor cerebri). Ophthalmology. 1998, 105:23132317. 10.1016/S0161-6420(98)91234-9

43. Sinclair AJ, Walker EA, Burdon MA, et al.: Cerebrospinal fluid corticosteroid levels and cortisol metabolism in patients with idiopathic intracranial hypertension: a link between 11beta-HSD1 and intracranial pressure regulation?. J Clin Endocrinol Metab. 2010, 95:5348-56. 10.1210/jc.2010-0729

44. Markey K, Mitchell J, Botfield H, et al.: 11ß-hydroxysteroid dehydrogenase type 1 inhibition in idiopathic intracranial hypertension: a double-blind randomized controlled trial. Brain Commun. 2020, 2 :fcz050. 10.1093/braincomms/fcz050

45. Simpson ER, Merrill JC, Hollub AJ, Graham-Lorence S, Mendelson CR: Regulation of estrogen biosynthesis by human adipose cells. Endocr Rev. 1989, 10:136-48. 10.1210/edrv-10-2-136

46. O'Reilly MW, Westgate CS, Hornby C, et al.: A unique androgen excess signature in idiopathic intracranial hypertension is linked to cerebrospinal fluid dynamics. JCI Insight. 2019, 4:e125348. 10.1172/jci.insight. 125348

47. Hayes KC, McCombs HL, Faherty TP: The fine structure of vitamin A deficiency. II. Arachnoid granulations and CSF pressure. Brain. 1971, 94:213-24. 10.1093/brain/94.2.213

48. Stenger MB, Laurie SS, Sadda SR, Sadun AA, Macias BR, Huang AS: Focus on the optic nerve head in spaceflight-associated neuro-ocular syndrome. Ophthalmology. 2019, 126:1604-1606. 10.1016/J.OPHTHA.2019.09.009

49. Hofman P, Hoyng P, vanderWerf F, Vrensen GF, Schlingemann RO: Lack of blood-brain barrier properties in microvessels of the prelaminar optic nerve head. Invest Ophthalmol Vis Sci. 2001, 42:895-901.

50. Wostyn P, De Winne F, Stern C, De Deyn PP: Dilated prelaminar paravascular spaces as a possible mechanism for optic disc edema in astronauts. Aerosp Med Hum Perform. 2018, 89:1089-1091. 10.3357/AMHP.5095.2018 